\title{
On the nonlocal Fisher-KPP equation: steady states, spreading speed and global bounds
}

\author{
François Hamel* $\quad$ Lenya Ryzhik ${ }^{\dagger}$
}

\begin{abstract}
We consider the Fisher-KPP equation with a non-local interaction term. We establish a condition on the interaction that allows for existence of non-constant periodic solutions, and prove uniform upper bounds for the solutions of the Cauchy problem, as well as upper and lower bounds on the spreading rate of the solutions with compactly supported initial data.
\end{abstract}

\section{Introduction and main results}

Reaction-diffusions equations of the form

$$
u_{t}=u_{x x}+\mu u(1-\phi * u), \quad t>0, \quad x \in \mathbb{R}
$$

model the non-local interaction and competition between species. Here, $u(t, x)$ is a population density, and the independent variable $x$ may be either a spatial location, a trait, or a mutation of the species. This equation is a generalization of the classical (local) Fisher-KPP (for KolmogorovPetrovsky-Piskunov) [10, 15] equation

$$
u_{t}=u_{x x}+\mu u(1-u) .
$$

The parameter $\mu>0$ in both cases denotes the strength of the competition. The nonnegative convolution kernel $\phi \in L^{1}(\mathbb{R})$ in (1.1) models the species interaction. We assume that it satisfies the following properties:

$$
\phi(x) \geq 0 \text { for all } x \in \mathbb{R}, \quad \underset{(-\sigma, \sigma)}{\operatorname{essinf}} \phi>0 \text { for some } \sigma>0, \text { and } \int_{\mathbb{R}} \phi=1 .
$$

This equation arises, in particular, in ecology, and adaptive dynamics [11, 12, 13, but also in essentially any area where the local Fisher-KPP equation (1.2) appears, as interactions are rarely fully local, and often occur on scales that are comparable to the diffusion scale, making the non-local equation a more realistic model.

\footnotetext{
*Aix Marseille Université, CNRS, Centrale Marseille, LATP, UMR 7353, 13453 Marseille, France, and Institut Universitaire de France

${ }^{\dagger}$ Department of Mathematics, Stanford University, Stanford, CA 94305, USA
} 


\section{Qualitative behavior of traveling waves and numerical solutions}

While the behavior of the solutions of the local equation (1.2) has been very extensively studied, much less is known about solutions of (1.1). Numerical simulations [6, 12, 13] indicate the following behavior of solutions: first, for small $\mu$ solutions of the non-local equation behave "exactly like solutions of the local equation". More precisely, numerics shows that when $\mu$ is small, the only non-negative bounded steady states for (1.1) are $u \equiv 0$ and $u \equiv 1$, and this equation admits monotonic traveling waves of the form

$$
u(t, x)=U(x-c t), \quad U(-\infty)=1, \quad U(+\infty)=0, \quad U \text { is decreasing, }
$$

that are asymptotically stable for the solutions of the Cauchy problem with front-like initial data. On the other hand, when $\mu$ is sufficiently large, the qualitative behavior of numerical solutions for (1.1) and (1.2) is very different: first, non-constant bounded steady solutions of (1.1) may exist. As far as traveling waves are concerned, when $\mu$ is large, numerical computations show that non-monotonic traveling waves connecting 1 to 0 may exist, as well as non-monotonic pulsating waves

$$
u(t, x)=U(x-c t, x), \quad U(-\infty, x)>0, \quad U(+\infty, x)=0, \quad U \text { is periodic in } x,
$$

connecting non-constant steady states to $u \equiv 0$. Moreover, the long time limit of the solutions of the Cauchy problem may be either non-monotonic traveling waves, pulsating waves or wave-trains

$$
u(t, x)=U(x-c t), \quad U \text { is periodic, }
$$

and not the monotonic traveling waves (see [6, 12, 13], and also [2] for a related model). To the best of our knowledge, so far, these numerical observations have not been proved rigorously.

When $\mu$ is sufficiently large or when $\phi$ is sufficiently far from a Dirac mass at 0 , the steady solution $u \equiv 1$ becomes unstable with respect to some spatially periodic perturbations, provided that the Fourier transform $\widehat{\phi}(\xi)$ is not non-negative everywhere. This, heuristically, leads to existence of the non-constant periodic steady states or wave trains that were observed numerically [12, 13] (see also [8, 14] for a bifurcation analysis and numerics on a related equation). Quite surprisingly, it was shown numerically in [16] that even when the state $u \equiv 1$ is unstable, there exist traveling wave solutions (which are themselves not stable) that connect $u \equiv 1$ to $u \equiv 0$. It has been proved in [6] that when $\widehat{\phi}(\xi) \geq 0$ for all $\xi \in \mathbb{R}$, so that $u \equiv 1$ is stable, and $\mu$ is sufficiently large, monotonic traveling waves connecting $u \equiv 0$ and $u \equiv 1$ do not exist, but there exists a non-monotonic traveling wave connecting these two states. We also point out that explicit examples of various wave-trains have been recently constructed in [17].

As far as monotone traveling waves for (1.1) are concerned, Fang and Zhao 9] have proved that for all $c \geq 2$, there exists $\mu_{c}>0$, so that (1.1) admits a monotonic traveling wave if and only if $\mu \in\left(0, \mu_{c}\right)$ - see also [13, 18]. It has been also shown in [1] that traveling waves with large speeds necessarily converge to 1 as $x-c t \rightarrow-\infty$.

Essentially nothing is known about the long time behavior of solutions of the Cauchy problem for (1.1), in particular, whether the aforementioned traveling waves are stable. The main mathematical difficulty in studying this question is that unlike for (1.2), solutions of (1.1) do not obey the maximum principle or the comparison principle, making the use of many technical tools impossible. 


\section{Non-trivial steady states}

We study here existence of nonnegative steady bounded solutions $u=u(x)$ of (1.1):

$$
u^{\prime \prime}(x)+\mu u(x)(1-(\phi * u)(x))=0, \quad x \in \mathbb{R} .
$$

Solutions are understood in the classical sense $C^{2}(\mathbb{R})$. Notice that, for any such solution $u$, (1.4) can be written as

$$
u^{\prime \prime}+c u=0 \text { in } \mathbb{R},
$$

where $c=\mu(1-\phi * u)$ is continuous and bounded in $\mathbb{R}$. Hence, for any nonnegative bounded solution $u$ of (1.4), either $u=0$ in $\mathbb{R}$ or $u>0$ in $\mathbb{R}$, from the strong maximum principle. The constant functions $u=0$ and $u=1$ solve (1.4) and they are the only constant functions solving (1.4), for every $\mu>0$. The following theorem gives a sufficient condition for other nontrivial periodic steady states to exist when $\mu$ is sufficiently large. We denote by $\widehat{\phi}$ the Fourier transform of the function $\phi$ :

$$
\widehat{\phi}(\xi)=\int_{\mathbb{R}} \phi(x) e^{-2 i \pi \xi x} d x \text { for all } \xi \in \mathbb{R} .
$$

Theorem 1.1 Assume that $\phi$ is even and that there are $L \in(0,+\infty)$ and $k_{0} \in \mathbb{N}$ such that

$$
\widehat{\phi}\left(\frac{k_{0}}{L}\right)<0 \text { and } \widehat{\phi}\left(\frac{k}{L}\right) \geq 0 \text { for all } k \in \mathbb{N} \backslash\left\{k_{0}\right\} \text {. }
$$

Then there is $\mu^{*}>0$ such that, for every $\mu>\mu^{*}$, the problem (1.4) has a non-constant positive $L$-periodic solution $u$.

A sufficient condition for (1.5) is that there exists $\xi^{*}>0$ such that $\widehat{\phi} \geq 0$ on $\left[\xi^{*},+\infty\right)$ and $\widehat{\phi}<0$ in a left neighborhood of $\xi^{*}$. In that case, we can take $L>0$ such that $1 / L<\xi^{*}<2 / L$ and $\widehat{\phi}(1 / L)<0$, that is (1.5) holds with $k_{0}=1$. For instance, consider $\beta>1$ large enough so that

$$
c_{\beta}:=1-1 / \sqrt{\beta}+1 / \beta>0,
$$

and define

$$
\phi_{\beta}(x)=\frac{1}{c_{\beta} \sqrt{\pi}}\left(e^{-x^{2}}-e^{-\beta x^{2}}+e^{-\beta^{2} x^{2}}\right) .
$$

The function $\phi_{\beta}$ is positive in $\mathbb{R}$ and its $L^{1}(\mathbb{R})$ norm is equal to 1 . Furthermore, the function $\widehat{\phi}_{\beta}$ is such that

$$
\widehat{\phi}_{\beta}(\sqrt{\beta})=\frac{1}{c_{\beta}}\left(e^{-\beta \pi^{2}}-\frac{e^{-\pi^{2}}}{\sqrt{\beta}}+\frac{e^{-\pi^{2} / \beta}}{\beta}\right) \sim-\frac{e^{-\pi^{2}}}{\sqrt{\beta}}<0 \text { as } \beta \rightarrow+\infty,
$$

while $\widehat{\phi}_{\beta}(\xi) \sim\left(c_{\beta} \beta\right)^{-1} e^{-\pi^{2} \xi^{2} / \beta^{2}}>0$ as $\xi \rightarrow+\infty$ for every fixed $\beta>1$ such that $c_{\beta}>0$. As a consequence, the function $\phi_{\beta}$ satisfies the assumptions of Theorem 1.1 for $\beta>1$ large enough. 


\section{Bounds for the solutions of the Cauchy problem}

We also consider solutions of the Cauchy problem

$$
\left\{\begin{array}{l}
u_{t}=u_{x x}+\mu u(1-\phi * u), \quad t>0, \quad x \in \mathbb{R}, \\
u(0, \cdot)=u_{0},
\end{array}\right.
$$

with a non-negative initial condition $u_{0} \in L^{\infty}(\mathbb{R}), u_{0} \geq 0$. Solutions of the corresponding Cauchy problem for the local Fisher-KPP equation (1.2) satisfy a trivial upper bound

$$
u(t, x) \leq \max \left(1,\left\|u_{0}\right\|_{L^{\infty}}\right)
$$

However, as the maximum principle does not hold for (1.6), such bound needs not hold for the non-local problem. We prove here the following result.

Theorem 1.2 For every $\mu>0$ and for every nonnegative initial condition $u_{0} \in L^{\infty}(\mathbb{R})$, the solution $u$ of (1.6) exists and is globally bounded in time, that is there exists $M>0$ such that

$$
0 \leq u(t, x) \leq M \text { for all } t>0 \text { and } x \in \mathbb{R}
$$

In Theorem 1.2, the condition that $\phi \geq 0$ belongs to $L^{1}(\mathbb{R})$ with unit mass could likely be replaced by the assumption that $\phi$ is a nonnegative Radon probability measure. On the other hand, the condition

$$
\operatorname{ess} \inf _{(-\sigma, \sigma)} \phi>0
$$

for some $\sigma>0$ plays a crucial role in Theorem 1.2. Without it, the conclusion fails in general. Let us illustrate this with the following example. Let $\mu$ and $L$ be two positive real numbers such that $\mu>\pi^{2} / L^{2}$ and consider (1.6) with

$$
\phi=\frac{1}{2}\left(\delta_{-L}+\delta_{L}\right)
$$

where $\delta_{ \pm L}$ denote the Dirac masses at the points $\pm L$. Assume that the initial data $u_{0}$ is $2 L$ periodic. By uniqueness, the function $u(t, \cdot)$ is $2 L$-periodic for every $t>0$ and it obeys

$$
\begin{aligned}
u_{t}(t, x) & =u_{x x}(t, x)+\mu u(t, x)\left(1-\frac{u(t, x-L)+u(t, x+L)}{2}\right) \\
& =u_{x x}(t, x)+\mu u(t, x)(1-u(t, x+L)),
\end{aligned}
$$

for every $(t, x) \in(0,+\infty) \times \mathbb{R}$. Set $v(t, x)=u(t, x+L)$ and $w(t, x)=u(t, x)-v(t, x)$. The functions $u, v$ and $w$ satisfy

$$
\begin{aligned}
& u_{t}=u_{x x}+\mu u(1-v), \\
& v_{t}=v_{x x}+\mu v(1-u), \\
& w_{t}=w_{x x}+\mu w,
\end{aligned}
$$

in $(0,+\infty) \times \mathbb{R}$. Therefore, if for instance one chooses

$$
u_{0}(x)=1+\rho \cos \left(\frac{\pi x}{L}\right)
$$


for some $0<\rho<1$, then $w(0, x)=2 \rho \cos (\pi x / L)$ and

$$
w(t, x)=2 \rho \cos \left(\frac{\pi x}{L}\right) e^{\left(\mu-\pi^{2} / L^{2}\right) t},
$$

for every $t>0$ and $x \in \mathbb{R}$. Since $\mu>\pi^{2} / L^{2}$, one gets

$$
\|w(t, \cdot)\|_{L^{\infty}(\mathbb{R})} \rightarrow+\infty \text { as } t \rightarrow+\infty .
$$

However, we have

$$
\|u(t, \cdot)\|_{L^{\infty}(\mathbb{R})}=\|v(t, \cdot)\|_{L^{\infty}(\mathbb{R})},
$$

and

$$
\|w(t, \cdot)\|_{L^{\infty}(\mathbb{R})} \leq\|u(t, \cdot)\|_{L^{\infty}(\mathbb{R})}+\|v(t, \cdot)\|_{L^{\infty}(\mathbb{R})}=2\|u(t, \cdot)\|_{L^{\infty}(\mathbb{R})},
$$

for every $t>0$. Finally, one concludes that $\|u(t, \cdot)\|_{L^{\infty}(\mathbb{R})} \rightarrow+\infty$ as $t \rightarrow+\infty$.

\section{Spreading rate for compactly supported initial conditions}

Finally, we establish the following result for the spreading rate for solutions of the Cauchy problem with compactly supported initial data.

Theorem 1.3 Let $u$ be the solution of the Cauchy problem (1.6) with a nonnegative initial condition $u_{0} \in L^{\infty}(\mathbb{R})$ such that $u_{0} \not \equiv 0$. Then

$$
\liminf _{t \rightarrow+\infty}\left(\min _{|x| \leq c t} u(t, x)\right)>0 \text { for all } 0 \leq c<2 \sqrt{\mu}
$$

Furthermore, if $u_{0}$ is compactly supported, then

$$
\lim _{t \rightarrow+\infty}\left(\max _{|x| \geq c t} u(t, x)\right)=0 \text { for all } c>2 \sqrt{\mu} .
$$

Similar bounds for the spreading rate for the solutions of the local Fisher-KPP equation (1.2) are well known [3]. And, indeed, the upper bound (1.8) on the spreading rate follows immediately from the comparison of the solutions of (1.6) and the solutions of the linear problem

$$
v_{t}=v_{x x}+\mu v
$$

On the other hand, the lack of the maximum principle for (1.6) makes the proof of the lower bound (1.7) very different from that for the local equation (1.2). The idea is, roughly, as follows. If $u(t, x)$ is small behind the location $x(t)=2 \sqrt{\mu} t$ then it should be small on a sufficiently large region to make also the convolution $\phi * u$ small. In that case, however, $u(t, x)$ behaves, approximately, as a solution of the linearized equation (1.9). These solutions, however, propagate with the speed $c_{*}=2 \sqrt{\mu}$, leading to a contradiction.

The paper is organized as follows. Section 2 contains the proof of Theorem 1.1. while Theorems 1.2 and 1.3 are proved in Sections 3 and 4 , respectively.

Acknowledgment. The research leading to these results has received funding from the French ANR within the project PREFERED and from the European Research Council under the 
European Union's Seventh Framework Programme (FP/2007-2013) / ERC Grant Agreement n.321186 - ReaDi - Reaction-Diffusion Equations, Propagation and Modelling. Part of this work was also carried out during visits by F. Hamel to the Departments of Mathematics of the University of California, Berkeley and of Stanford University, the hospitality of which is thankfully acknowledged. This work was also supported by the NSF grants DMS-0908507 and DMS-115893. The authors are grateful to G. Nadin and B. Perthame for valuable discussions during the preparation of this work.

\section{Existence of non-constant periodic steady states}

This section contains the proof of Theorem 1.1. The general strategy is to apply a topological degree argument. We first prove that, if $\mu>0$ is small enough, the constant solution $u \equiv 1$ is linearly stable and is the only bounded positive solution of (1.4). Next, we show that, under the assumptions of the theorem, if $\mu>0$ is large enough, the constant $u \equiv 1$ is an isolated (in the $L^{\infty}$-sense) unstable steady solution with one unstable direction in the class of $L$-periodic even functions. Together with some a priori uniform estimates, this will allow us conclude that $u \equiv 1$ is not the only positive solution of (1.4) for large values of the parameter $\mu$. Throughout this section, we assume that the convolution kernel $\phi$ is a nonnegative $L^{1}(\mathbb{R})$ function satisfying (1.3). The additional assumption (1.5) and the evenness of $\phi$ will be used only in some steps of the proof.

\section{Uniform bounds for solutions}

We first establish uniform pointwise upper and lower bounds for all positive bounded solutions of (1.4), when the parameter $\mu$ ranges between two fixed positive constants.

Lemma 2.1 For every $0<a \leq b<+\infty$, there is a positive constant $m$ which depends on $\phi, a$ and $b$ and there is a positive constant $M$ which depends on $\phi$ and $b$, such that, for every $\mu \in[a, b]$ and every positive bounded solution $u$ of (1.4), there holds

$$
0<m \leq u(x) \leq M \text { for all } x \in \mathbb{R}
$$

Proof. Similar lower and upper bounds were proved for a fixed value of $\mu>0$ in Lemma 2.1 and Proposition 2.3 of [6], under the additional assumption that the convolution kernel $\phi$ is of class $C^{1}$ and has a bounded second moment. Lemma 2.1 of the present paper can be viewed as an extension of the aforementioned results. Furthermore, the proof of the upper bound is slightly different from that of Proposition 2.3 of [6].

We begin with the proof of the upper bound, that is the existence of a constant $M$ in (2.1), independently of $\mu \in[a, b]$ and of $u>0$ solving (1.4). Let $\sigma>0$ be as in (1.3), and $\delta$ be any fixed real number such that

$$
0<\delta<\min \left(\frac{\pi}{2 \sqrt{b}}, \sigma\right) \text {. }
$$

Finally, let $\eta>0$ be such that

$$
\phi(x) \geq \eta>0 \text { for almost every } x \in(-\delta, \delta) .
$$


From the choice of $\delta$, we know that the lowest eigenvalue of the operator

$$
\varphi \mapsto-\varphi^{\prime \prime}-b \varphi
$$

in $(-\delta, \delta)$ with the Dirichlet boundary condition at $\pm \delta$, which is equal to $\pi^{2} /\left(4 \delta^{2}\right)-b$, is positive. Therefore, the weak maximum principle for this operator holds in the interval $(-\delta, \delta)$ (see [7]) and in any subinterval, and there is a unique $C^{2}([-\delta, \delta])$ solution $\bar{u}$ of the boundary value problem

$$
\bar{u}^{\prime \prime}+b \bar{u}=0 \text { in }[-\delta, \delta] \text { and } \bar{u}( \pm \delta)=\frac{1}{\delta \eta} .
$$

Furthermore, since the constant $1 /(\delta \eta)$ is a subsolution for the above equation, the weak maximum principle yields

$$
\bar{u} \geq \frac{1}{\delta \eta} \text { in }[-\delta, \delta]
$$

Notice that $\delta$ depends only on $\phi$ and $b$, that $\eta$ depends only on $\phi$ and $\delta$ and that $\bar{u}$ depends only on $b, \delta$ and $\eta$, that is $\bar{u}$ depends only on $\phi$ and $b$.

Let now $\mu$ be any real number in $[a, b], u$ be any positive bounded solution of (1.4) and set

$$
\rho_{u}=\sup _{\mathbb{R}} u>0 .
$$

Since (1.4) implies that $u^{\prime \prime}$ is bounded, we know that $u^{\prime}$ is also bounded. By differentiating (1.4), we see that the $k$ th-order derivative $u^{(k)}$ of $u$ exists and is bounded, for every $k \in \mathbb{N}$. Furthermore, there is a sequence $\left(x_{n}\right)_{n \in \mathbb{N}}$ of real numbers such that

$$
u\left(x_{n}\right) \rightarrow \rho_{u} \text { as } n \rightarrow+\infty
$$

Consider the translates

$$
u_{n}(x)=u\left(x+x_{n}\right) \text { for } n \in \mathbb{N} \text { and } x \in \mathbb{R} .
$$

Up to extraction of a subsequence, the functions $u_{n}$ converge in (at least) $C_{\text {loc }}^{2}(\mathbb{R})$ to a $C^{\infty}(\mathbb{R})$ solution $u_{\infty}$ of (1.4) such that $0 \leq u_{\infty} \leq \rho_{u}$ in $\mathbb{R}$ and $u_{\infty}(0)=\rho_{u}>0$. Therefore, we have $u_{\infty}^{\prime \prime}(0) \leq 0$, whence

$$
\int_{\mathbb{R}} \phi(y) u_{\infty}(-y) d y=\left(\phi * u_{\infty}\right)(0) \leq 1
$$

Since both $\phi$ and $u_{\infty}$ are nonnegative, one gets that

$$
\int_{-\delta}^{0} \phi(y) u_{\infty}(-y) d y \leq 1 \text { and } \int_{0}^{\delta} \phi(y) u_{\infty}(-y) d y \leq 1 .
$$

Here, $\delta$ is defined in (2.2). On the other hand, $\phi \geq \eta>0$ almost everywhere in $(-\delta, \delta)$, due to (2.3). One infers the existence of some real numbers $y_{ \pm}$such that

$$
-\delta \leq y_{-}<0<y_{+} \leq \delta \text { and } u_{\infty}\left(y_{ \pm}\right) \leq \frac{1}{\delta \eta}
$$

On the other hand, the nonnegative function $u_{\infty}$ satisfies

$$
0=u_{\infty}^{\prime \prime}+\mu u_{\infty}\left(1-\phi * u_{\infty}\right) \leq u_{\infty}^{\prime \prime}+\mu u_{\infty} \leq u_{\infty}^{\prime \prime}+b u_{\infty} \text { in } \mathbb{R} .
$$


That is, $u_{\infty}$ is a sub-solution to the equation (2.5) satisfied by the function $\bar{u}$. Therefore, the lower bound (2.6) on $\bar{u}$, and the upper bound (2.7) for $u_{\infty}$ at $y=y_{ \pm}$, together with the maximum principle for the operator (2.4), imply that

$$
u_{\infty} \leq \bar{u} \text { in }\left[y_{-}, y_{+}\right]
$$

whence $\rho_{u}=u_{\infty}(0) \leq \bar{u}(0)$. Since $\bar{u}(0)$ depends only on $\phi$ and $b$ and does not depend on $\mu$ nor on $u$, the upper bound in Lemma 2.1 is thereby proved.

We now turn to the proof of the lower bound in Lemma 2.1, that is, the existence of a positive constant $m$, independent of $\mu \in[a, b]$ so that any bounded solution $u>0$ of (1.4) is bounded from below by $m$. Assume that there is no such constant $m>0$. Then, there exist a sequence $\left(\mu_{n}\right)_{n \in \mathbb{N}}$ of real numbers in the interval $[a, b]$, a sequence $\left(u_{n}\right)_{n \in \mathbb{N}}$ of positive bounded solutions of (1.4) with $\mu=\mu_{n}$, and a sequence $\left(x_{n}\right)_{n \in \mathbb{N}}$ of real numbers such that

$$
u_{n}\left(x_{n}\right) \rightarrow 0 \text { as } n \rightarrow+\infty \text {. }
$$

Each function $u_{n}$ is positive, and, from the already proved part of the present lemma, there is a constant $M>0$ such that $u_{n} \leq M$ in $\mathbb{R}$ for all $n \in \mathbb{N}$. As a consequence, the sequence $\left(u_{n}^{\prime \prime}\right)_{n \in \mathbb{N}}$ is bounded in $L^{\infty}(\mathbb{R})$, as are $\left(u_{n}^{\prime}\right)_{n \in \mathbb{N}}$ and $\left(u_{n}^{(k)}\right)_{n \in \mathbb{N}}$ for every $k \in \mathbb{N}$ by immediate induction. Again, we consider the shifts

$$
v_{n}(x)=u_{n}\left(x+x_{n}\right) .
$$

Up to extraction of a subsequence, one can assume that $\mu_{n} \rightarrow \mu \in[a, b]$ as $n \rightarrow+\infty$ and that the sequence $\left(v_{n}\right)_{n \in \mathbb{N}}$ converges as $n \rightarrow+\infty$ in (at least) $C_{l o c}^{2}(\mathbb{R})$ to a $C^{\infty}(\mathbb{R})$ solution $v_{\infty}$ of (1.4) such that $0 \leq v_{\infty} \leq M$ in $\mathbb{R}$ and $v_{\infty}(0)=0$. The function $v_{\infty}$ satisfies

$$
v_{\infty}^{\prime \prime}+c v_{\infty}=0 \text { in } \mathbb{R}
$$

where

$$
c=\mu\left(1-\phi * v_{\infty}\right)
$$

is a bounded continuous function. The strong maximum principle implies that $v_{\infty} \equiv 0$ in $\mathbb{R}$, that is $v_{n} \rightarrow 0$ as $n \rightarrow+\infty$ (at least) locally uniformly in $\mathbb{R}$. Since $\phi$ is a nonnegative $L^{1}(\mathbb{R})$ function, and all functions $v_{n}$ satisfy $0<v_{n} \leq M$ in $\mathbb{R}$, it follows that

$$
\phi * v_{n} \rightarrow 0 \text { as } n \rightarrow+\infty \text { locally uniformly in } \mathbb{R} \text {. }
$$

In particular, for every $R>0$, there is $N \in \mathbb{N}$ such that $\phi * v_{n} \leq 1 / 2$ in $[-R, R]$ for every $n \geq N$, whence

$$
0=v_{n}^{\prime \prime}+\mu_{n} v_{n}\left(1-\phi * v_{n}\right) \geq v_{n}^{\prime \prime}+\frac{\mu_{n} v_{n}}{2} \geq v_{n}^{\prime \prime}+\frac{a v_{n}}{2} \text { in }[-R, R]
$$

for every $n \geq N$. In other words, all functions $v_{n}$ (for $n \geq N$ ) are supersolutions of the elliptic operator

$$
\varphi \mapsto-\varphi^{\prime \prime}-(a / 2) \varphi \text { in }[-R, R]
$$

Since the functions $v_{n}$ are all positive in $[-R, R]$, it follows from [7] that the lowest eigenvalue of this operator with Dirichlet boundary condition at the points $\pm R$ is positive, that is

$$
\frac{\pi^{2}}{4 R^{2}}-\frac{a}{2}>0
$$

Since this holds for every $R>0$, one gets a contradiction as $R \rightarrow+\infty$. Hence, the sequences $\left(\mu_{n}\right)_{n \in \mathbb{N}},\left(u_{n}\right)_{n \in \mathbb{N}}$ and $\left(x_{n}\right)_{n \in \mathbb{N}}$ as above can not exist. That yields the existence of $m$ in (2.1) and completes the proof of Lemma 2.1. 


\section{Non-existence of non-trivial solutions for small $\mu$}

We proceed with the proof of Theorem 1.1. Let now $L>0$ and $k_{0} \in \mathbb{N}$ be as in the assumption (1.5) of Theorem 1.1. We also assume from now on that the convolution kernel $\phi$ is even. Let $\alpha$ be any fixed real number in the interval $(0,1)$, and let $X$ be the Banach space of even $L$-periodic functions of class $C^{0, \alpha}(\mathbb{R})$, equipped with the norm

$$
\|u\|_{X}=\|u\|_{L^{\infty}(\mathbb{R})}+\sup _{x \neq y \in \mathbb{R}} \frac{|u(x)-u(y)|}{|x-y|^{\alpha}} .
$$

For every $\mu>0$, let $T_{\mu}: X \rightarrow X$ be an operator defined as follows: for every $u \in X$, $v:=T_{\mu}(u) \in X$ is the unique classical solution of

$$
-v^{\prime \prime}+v=u+\mu u(1-\phi * u) \text { in } \mathbb{R} .
$$

From the Lax-Milgram theorem and the standard interior elliptic estimates, $T_{\mu}(u)$ is well defined and belongs to $X \cap C^{2, \alpha}(\mathbb{R})$ for every $u \in X$. The operator $T_{\mu}$ is continuous, and compact in the sense that it maps bounded subsets of $X$ into subsets of $X$ with compact closure. Furthermore, again from the elliptic estimates, the map $\mu \mapsto T_{\mu}$ is continuous locally uniformly in $X$, in the sense that, for every $\mu>0$, every sequence $\left(\mu_{n}\right)_{n \in \mathbb{N}}$ converging to $\mu$ and every bounded subset $B$ of $X$, one has

$$
\sup _{u \in B}\left\|T_{\mu_{n}}(u)-T_{\mu}(u)\right\|_{X} \rightarrow 0 \text { as } n \rightarrow+\infty .
$$

Notice also that the constant function $1 \in X$ is a fixed point of $T_{\mu}$, that is, $T_{\mu}(1)=1$, for every $\mu>0$. In order to get the conclusion of Theorem 1.1, our goal is to show that, when $\mu>0$ is large enough, there are non-constant positive fixed points of $T_{\mu}$ in $X$, that is, positive non-constant solutions $u \in X$ of

$$
u-T_{\mu}(u)=0 .
$$

We will do so by evaluating the Leray-Schauder topological degree of the map $I-T_{\mu}$ at the point 0 in some suitable open subsets of $X$ and by using a homotopy argument from small values of the parameter $\mu$ to large values of $\mu$. Here, $I$ denotes the identity map.

The first step is to show that the constant function $u \equiv 1$ is the only positive fixed point of $T_{\mu}$ in $X$ when $\mu>0$ is small enough.

Lemma 2.2 There is $\mu>0$ such that, for every $\mu \in(0, \mu)$, the constant function $u \equiv 1$ is the only positive fixed point of $T_{\mu}$ in $X$, that is the only positive solution of (1.4) in $X$.

Proof. In Theorem 1.1 of [6], the stronger conclusion that the constant function 1 is the only positive bounded solution of (1.4) for small $\mu>0$ was proved, under some slightly stronger smoothness assumptions on $\phi$. In the present Lemma 2.2, uniqueness holds with slightly different assumptions on $\phi$, but is shown here only in the space $X$, so the proof is shorter. It is presented for the sake of completeness.

Assume that the conclusion is not true. Then there is a sequence $\left(\mu_{n}\right)_{n \in \mathbb{N}}$ of positive real numbers such that $\mu_{n} \rightarrow 0$ as $n \rightarrow+\infty$, and a sequence $\left(u_{n}\right)_{n \in \mathbb{N}}$ in $X$ of solutions of (1.4) with $\mu=\mu_{n}$, such that $0<u_{n}$ in $\mathbb{R}$ and $u_{n} \not \equiv 1$. From Lemma 2.1, there is a constant $M$ such that

$$
0<u_{n} \leq M \text { in } \mathbb{R}
$$


for every $n \in \mathbb{N}$. If $\max _{\mathbb{R}} u_{n} \leq 1$, then $\phi * u_{n} \leq 1$ and $u_{n}^{\prime \prime} \leq 0$ in $\mathbb{R}$, implying that $u_{n}$ is a positive constant, which would then have to be equal to 1 by (1.4). Therefore,

$$
\max _{\mathbb{R}} u_{n}>1 \text { for every } n \in \mathbb{N} \text {. }
$$

As done in the proof of Lemma 2.1, it follows from (1.4) that the $L$-periodic functions $u_{n}$ are bounded in $C^{k}(\mathbb{R})$ for every $k \in \mathbb{N}$, and therefore converge, as $n \rightarrow+\infty$, up to extraction of a subsequence, to a $C^{\infty}(\mathbb{R})$ solution $u_{\infty}$ of

$$
u_{\infty}^{\prime \prime}=0
$$

such that $0 \leq u_{\infty} \leq M$ in $\mathbb{R}$. As a consequence, the function $u_{\infty}$ is constant and this constant is such that $1 \leq u_{\infty} \leq M$ due to (2.8). If $u_{\infty}>1$, then, as all $u_{n}$ are $L$-periodic, we have $u_{n}>1$ in $\mathbb{R}$ for $n$ large enough, whence $\phi * u_{n}>1$ and $u_{n}^{\prime \prime}>0$ in $\mathbb{R}$ for $n$ large enough, which is impossible since all $u_{n}$ are bounded. We conclude that

$$
u_{\infty} \equiv 1
$$

Next, write $u_{n}=1+v_{n}$ and $w_{n}=v_{n} /\left\|v_{n}\right\|_{L^{\infty}(\mathbb{R})}$. Since $\max _{\mathbb{R}} u_{n}>1$, one has $\max _{\mathbb{R}} v_{n}>0$ and $w_{n}$ is well defined, for every $n \in \mathbb{N}$. Furthermore, $v_{n} \rightarrow 0$ as $n \rightarrow+\infty$ in $C^{k}(\mathbb{R})$ for every $k \in \mathbb{N}$. The functions $w_{n}$ are $L$-periodic, with $\max _{\mathbb{R}} w_{n}>0$ and $\left\|w_{n}\right\|_{L^{\infty}(\mathbb{R})}=1$. They obey

$$
w_{n}^{\prime \prime}-\mu_{n} u_{n}\left(\phi * w_{n}\right)=0 \text { in } \mathbb{R} .
$$

From the standard elliptic estimates, we know that the functions $w_{n}$ converge as $n \rightarrow+\infty$, up to extraction of a subsequence, in $C^{k}(\mathbb{R})$ for every $k \in \mathbb{N}$, to a $C^{\infty}(\mathbb{R})$ solution $w_{\infty}$ of

$$
w_{\infty}^{\prime \prime}=0,
$$

such that $\max _{\mathbb{R}} w_{\infty} \geq 0$ and $\left\|w_{\infty}\right\|_{L^{\infty}(\mathbb{R})}=1$. It follows that $w_{\infty} \equiv 1$. For $n$ large enough, one gets that $w_{n}>0$ in $\mathbb{R}$, once again since $w_{n}$ are $L$-periodic, whence

$$
u_{n}=1+\left\|v_{n}\right\|_{L^{\infty}(\mathbb{R})} w_{n}>1 \text { in } \mathbb{R} .
$$

Finally, it follows that $\phi * u_{n}>1$ and $u_{n}^{\prime \prime}>0$ in $\mathbb{R}$ for $n$ large enough, which is impossible since $u_{n}$ is bounded. One has then reached a contradiction and the proof of Lemma 2.2 is thereby complete.

\section{Stability of the fixed points}

Let us now study the stability of the fixed point 1 of $T_{\mu}$ when $\mu>0$ is small or large. It follows from elementary calculations and standard elliptic estimates that $T_{\mu}$ is Fréchet-differentiable everywhere in $X$ and that the Fréchet-derivative $D T_{\mu}(1)$ at the point 1 is given as follows: for every $u \in X, w=D T_{\mu}(1)(u)$ is the unique solution of

$$
-w^{\prime \prime}+w=u-\mu \phi * u \text { in } \mathbb{R} .
$$

Notice that the linear operator $D T_{\mu}(1): X \rightarrow X$ is compact.

The next two lemmas are concerned with the stability of the fixed point 1 of $T_{\mu}$ when $\mu>0$ is small and when $\mu>0$ is large. 
Lemma $2.3($ Small $\mu)$ There is $\underline{\underline{\mu}}>0$ such that, for every $\mu \in(0, \underline{\underline{\mu}})$, all eigenvalues $\lambda$ of $D T_{\mu}(1)$ satisfy $|\lambda|<1$.

Proof. Let $\underline{\underline{\mu}}$ be given by

$$
\underline{\underline{\mu}}=\min \left(\frac{4 \pi^{2}}{L^{2}}, \frac{1}{2}\right)
$$

and let us check that the conclusion holds with this value $\underline{\underline{\mu}}$. To this end, let $\mu \in(0, \underline{\underline{\mu}})$ and let $u$ be an eigenvector of $D T_{\mu}(1)$ with the eigenvalue $\lambda$. The function $u \in X$ solves

$$
-(\lambda u)^{\prime \prime}+\lambda u=u-\mu \phi * u \text { in } \mathbb{R} .
$$

Let $\left(a_{k}\right)_{k \in \mathbb{N}}$ be the Fourier coefficients of $u$, defined by

$$
a_{k}=\frac{2}{L} \int_{0}^{L} u(x) \cos \left(\frac{2 \pi k x}{L}\right) d x, \quad k \in \mathbb{N} .
$$

The Fourier coefficients $b_{k}$ of $\phi * u$ are given by

$$
b_{k}=\frac{2}{L} \int_{0}^{L}(\phi * u)(x) \cos \left(\frac{2 \pi k x}{L}\right) d x=\widehat{\phi}\left(\frac{k}{L}\right) a_{k}, \quad k \in \mathbb{N},
$$

since $\phi$ is even and real-valued.

Since $u$ is even, $L$-periodic and does not vanish identically, there is $m_{0} \in \mathbb{N}$ such that $a_{m_{0}} \neq 0$. If $\lambda$ were equal to 0 , then, in particular, the $m_{0}$-th Fourier coefficient of the right-hand side of (2.9) would vanish, that is

$$
a_{m_{0}}-\mu \widehat{\phi}\left(\frac{m_{0}}{L}\right) a_{m_{0}}=0
$$

whence

$$
1-\mu \widehat{\phi}\left(m_{0} / L\right)=0
$$

as $a_{m_{0}} \neq 0$. Thus, we would have

$$
1=\mu \widehat{\phi}\left(m_{0} / L\right) \leq \underline{\underline{\mu}}\|\phi\|_{L^{1}(\mathbb{R})}=\underline{\underline{\mu}} \leq 1 / 2,
$$

which is impossible. As a consequence, $\lambda \neq 0$. It follows then from (2.9) that the function $u$ is actually of class $C^{\infty}(\mathbb{R})$ and that

$$
\left(\frac{4 \pi^{2} k^{2}}{L^{2}}+1\right) \lambda a_{k}=a_{k}-\mu \widehat{\phi}\left(\frac{k}{L}\right) a_{k} \text { for all } k \in \mathbb{N} .
$$

In particular, since $a_{m_{0}} \neq 0$, one gets that

$$
\lambda\left(\frac{4 \pi^{2} m_{0}^{2}}{L^{2}}+1\right)=1-\mu \widehat{\phi}\left(\frac{m_{0}}{L}\right) .
$$

If $m_{0}=0$, then $\lambda=1-\mu$, whence $\lambda \in(1-\underline{\underline{\mu}}, 1) \subset(1 / 2,1)$. If $m_{0} \geq 1$, then

$$
|\lambda|\left(\frac{4 \pi^{2} m_{0}^{2}}{L^{2}}+1\right)=\left|1-\mu \widehat{\phi}\left(\frac{m_{0}}{L}\right)\right|<1+\underline{\underline{\mu}} \leq 1+\frac{4 \pi^{2}}{L^{2}},
$$

whence $|\lambda|<1$. The proof of Lemma 2.3 is thereby complete. 
Lemma 2.4 (Large $\mu$ ) There is $\mu^{*}>0$ such that, for every $\mu>\mu^{*}$, the operator $D T_{\mu}(1)$ has an eigenvalue $\lambda_{\mu}>1$, with $\operatorname{dim} \operatorname{ker}\left(D T_{\mu}(1)-\lambda_{\mu} I\right)^{n}=1$ for all $n \geq 1$. Moreover, all other eigenvalues $\lambda$ of $D T_{\mu}(1)$ satisfy $\lambda<1$.

Proof. Recall that we assume that there exists $k_{0} \in \mathbb{N}$ such that $\widehat{\phi}\left(k_{0} / L\right)<0$ and $\widehat{\phi}(k / L) \geq 0$ for all $k \in \mathbb{N} \backslash\left\{k_{0}\right\}$. As $\widehat{\phi}(0)=1$, we have $k_{0} \geq 1$. Let $\mu_{*}>0$ be defined by

$$
\mu^{*}=\frac{4 \pi^{2} k_{0}^{2}}{L^{2}} \times \frac{1}{\left|\widehat{\phi}\left(\frac{k_{0}}{L}\right)\right|}>0
$$

and let us show that the conclusion of Lemma 2.4 holds with this choice of $\mu^{*}$. Let $\mu$ be any real number such that $\mu>\mu^{*}$, and set

$$
\lambda_{\mu}=\frac{1-\mu \widehat{\phi}\left(\frac{k_{0}}{L}\right)}{\frac{4 \pi^{2} k_{0}^{2}}{L^{2}}+1}>1
$$

by (2.11). The function $u_{\mu} \in X$ given by

$$
u_{\mu}(x)=\cos \left(\frac{2 \pi k_{0} x}{L}\right) \text { for every } x \in \mathbb{R},
$$

solves

$$
-\lambda_{\mu} u_{\mu}^{\prime \prime}+\lambda_{\mu} u_{\mu}=\left(1-\mu \widehat{\phi}\left(\frac{k_{0}}{L}\right)\right) u_{\mu}=u_{\mu}-\mu \phi * u_{\mu} \text { in } \mathbb{R} .
$$

In other words, $D T_{\mu}(1)\left(u_{\mu}\right)=\lambda_{\mu} u_{\mu}$, that is $u_{\mu}$ is an eigenvector of $D T_{\mu}(1)$ with the eigenvalue $\lambda_{\mu}>1$.

Moreover, if $u \in X$ satisfies $D T_{\mu}(1)(u)=\lambda_{\mu} u$, that is

$$
-\lambda_{\mu} u^{\prime \prime}+\lambda_{\mu} u=u-\mu \phi * u \text { in } \mathbb{R}
$$

then, since $\lambda_{\mu} \neq 0$, the function $u$ is actually of class $C^{\infty}(\mathbb{R})$ and its Fourier coefficients $a_{k}$ given by (2.10) satisfy

$$
\left(\frac{4 \pi^{2} k^{2}}{L^{2}}+1\right) \lambda_{\mu} a_{k}=\left(1-\mu \widehat{\phi}\left(\frac{k}{L}\right)\right) a_{k}
$$

for every $k \in \mathbb{N}$. For every $k \in \mathbb{N} \backslash\left\{k_{0}\right\}$, there holds

$$
\left(\frac{4 \pi^{2} k^{2}}{L^{2}}+1\right) \lambda_{\mu} \geq \lambda_{\mu}>1 \geq 1-\mu \widehat{\phi}\left(\frac{k}{L}\right)
$$

by the assumption (1.5). Therefore, $a_{k}=0$ for every $k \in \mathbb{N} \backslash\left\{k_{0}\right\}$. Since $u$ is even, it is then a multiple of the function $u_{\mu}(x)=\cos \left(2 \pi k_{0} x / L\right)$.

Next, let $u \in X$ be such that

$$
\left(D T_{\mu}(1)-\lambda_{\mu} I\right)^{2}(u)=0,
$$


that is $v=D T_{\mu}(1) u-\lambda_{\mu} u$ is in the eigenspace of $D T_{\mu}(1)$ with eigenvalue $\lambda_{\mu}$. From the previous paragraph, one knows that $v=\gamma u_{\mu}$ for some $\gamma \in \mathbb{R}$. In other words,

$$
D T_{\mu}(1)(u)=\lambda_{\mu} u+\gamma u_{\mu}
$$

In particular, the function $u$ is of class $C^{\infty}(\mathbb{R})$ and solves

$$
-\left(\lambda_{\mu} u+\gamma u_{\mu}\right)^{\prime \prime}+\left(\lambda_{\mu} u+\gamma u_{\mu}\right)=u-\mu \phi * u .
$$

Therefore, the Fourier coefficients $a_{k}$ of $u$, given by (2.10), satisfy (2.14) for every $k \in \mathbb{N} \backslash\left\{k_{0}\right\}$. As in the previous paragraph, it follows that $a_{k}=0$ for every $k \in \mathbb{N} \backslash\left\{k_{0}\right\}$, hence, $u$ is a multiple of the function $u_{\mu}$.

As a consequence, $\operatorname{dim} \operatorname{ker}\left(D T_{\mu}(1)-\lambda_{\mu} I\right)^{n}=1$ for every $n \geq 1$, and the algebraic and geometric multiplicity of the eigenvalue $\lambda_{\mu}$ of $D T_{\mu}(1)$ is equal to 1.

It only remains to show that the other eigenvalues of $D T_{\mu}(1)$ are all less than 1 . To do so, let $\lambda$ be any real number such that $\lambda \neq \lambda_{\mu}$ and $\lambda \geq 1$, and let $u \in X$ be such that $D T_{\mu}(1)(u)=\lambda u$. One has to prove that $u$ is necessarily equal to 0 . The function $u$ is of class $C^{\infty}(\mathbb{R})$, it solves (2.13) with the parameter $\lambda$ and its Fourier coefficients $a_{k}$ given by (2.10) satisfy (2.14) with the parameter $\lambda$, that is,

$$
\left(\frac{4 \pi^{2} k^{2}}{L^{2}}+1\right) \lambda a_{k}=\left(1-\mu \widehat{\phi}\left(\frac{k}{L}\right)\right) a_{k} \text { for every } k \in \mathbb{N} .
$$

Given the definition (2.12) of $\lambda_{\mu}$, and given that $\lambda \neq \lambda_{\mu}$, it follows that $a_{k_{0}}=0$. Furthermore, for $k=0$, one has $\lambda \geq 1>1-\mu=1-\mu \widehat{\phi}(0)$, whence $a_{0}=0$. Lastly, for every $k \in \mathbb{N} \backslash\left\{0, k_{0}\right\}$, there holds

$$
\left(\frac{4 \pi^{2} k^{2}}{L^{2}}+1\right) \lambda>\lambda \geq 1 \geq 1-\mu \widehat{\phi}\left(\frac{k}{L}\right)
$$

whence $a_{k}=0$. As a consequence, $a_{k}=0$ for every $k \in \mathbb{N}$, that is $\lambda$ cannot be an eigenvalue of $D T_{\mu}(1)$. That completes the proof of Lemma 2.4.

Remark 2.5 The fact that $X$ only contains even $L$-periodic functions forces the operator $D T_{\mu}(1)$, for large $\mu$, to have only one eigendirection associated to an eigenvalue larger than 1 . That will yield the explicit value, namely -1 , of the index of the map $I-T_{\mu}$ at 0 in a small neighborhood around the point $u=1$ and will finally lead to the existence of other (than $u=1$ ) fixed points of $T_{\mu}$ in $X$ for large $\mu$.

\section{End of the proof of Theorem 1.1}

Let $\underline{\mu}>0$ and $\underline{\underline{\mu}}>0$ be as in Lemmas 2.2 and 2.3 , and set

$$
\mu_{*}=\min (\underline{\mu}, \underline{\underline{\mu}})>0
$$

Let $\mu^{*}>0$ be as in Lemma 2.4. From Lemmas 2.3 and 2.4, it follows that $\mu_{*} \leq \mu^{*}$.

Let now $\mu_{0}$ and $\widetilde{\mu}$ be any two real numbers such that

$$
0<\mu_{0}<\mu_{*} \leq \mu^{*}<\widetilde{\mu}
$$


From Lemma 2.1, there are two positive constants $0<m \leq M$ such that every positive bounded solution $u$ of (1.4), with parameter $\mu \in\left[\mu_{0}, \widetilde{\mu}\right]$, satisfies

$$
0<m \leq u(x) \leq M \text { for all } x \in \mathbb{R}
$$

Notice that $u \equiv 1$ is a solution of (1.4) for every $\mu$, whence $0<m \leq 1 \leq M$.

Let $\Omega$ be the non-empty open bounded subset of $X$ defined by

$$
\Omega=\left\{u \in X, \frac{m}{2}<\min _{\mathbb{R}} u \leq \max _{\mathbb{R}} u<2 M\right\} .
$$

It follows from Lemma 2.1 and the choice of $m$ and $M$ that, for every $\mu \in\left[\mu_{0}, \widetilde{\mu}\right]$ and for every $u \in \partial \Omega$, there holds

$$
u-T_{\mu} u \neq 0,
$$

for otherwise $u$ would be a positive solution of (1.4) with the value $\mu$, whence $m \leq u \leq M$ in $\mathbb{R}$ and $u \notin \partial \Omega$. Therefore, for every $\mu \in\left[\mu_{0}, \widetilde{\mu}\right]$, the Leray-Schauder topological degree $\operatorname{deg}\left(I-T_{\mu}, \Omega, 0\right)$ of the map $I-T_{\mu}$ in the set $\bar{\Omega}$ at the point 0 is well defined. Furthermore, by the continuity of $T_{\mu}$ with respect to $\mu$ in the local uniform sense in $X$, this degree does not depend on $\mu \in\left[\mu_{0}, \widetilde{\mu}\right]$.

Let us now compute the degree. Lemmas 2.2 and 2.3 imply that $\operatorname{deg}\left(I-T_{\mu_{0}}, \Omega, 0\right)$ is equal to the index $\operatorname{ind}\left(I-T_{\mu_{0}}, 1,0\right)$ of $I-T_{\mu_{0}}$ at the point $u=1$, namely $\operatorname{ind}\left(I-T_{\mu_{0}}, 1,0\right)=1$. In other words, $\operatorname{deg}\left(I-T_{\mu_{0}}, \Omega, 0\right)=1$, whence

$$
\operatorname{deg}\left(I-T_{\widetilde{\mu}}, \Omega, 0\right)=1 .
$$

On the other hand, it follows from Lemma 2.4 that the operator $I-D T_{\widetilde{\mu}}(1)$ is one-to-one. Since $I-D T_{\widetilde{\mu}}(1)$ is a Fredholm operator of index 0 , it is invertible and the point $u=1$ is an isolated zero of $I-T_{\widetilde{\mu}}$ in $X$. Finally, $\operatorname{ind}\left(I-T_{\widetilde{\mu}}, 1,0\right)=-1$ since $I-D T_{\widetilde{\mu}}(1)$ has only one direction associated to a negative eigenvalue. In other words,

$$
\operatorname{deg}\left(I-T_{\widetilde{\mu}}, B_{\varepsilon}(1), 0\right)=\operatorname{ind}\left(I-T_{\widetilde{\mu}}, 1,0\right)=-1
$$

for $\varepsilon>0$ small enough, where $B_{\varepsilon}(1)$ denotes the open ball of center 1 and radius $\varepsilon$ in $X$. But

$$
\operatorname{deg}\left(I-T_{\widetilde{\mu}}, \Omega, 0\right)=\operatorname{deg}\left(I-T_{\widetilde{\mu}}, B_{\varepsilon}(1), 0\right)+\operatorname{deg}\left(I-T_{\widetilde{\mu}}, \Omega \backslash B_{\varepsilon}(1), 0\right),
$$

for $\varepsilon>0$ small enough. Together with (2.15) and (2.16), one finally concludes that, for $\varepsilon>0$ small enough,

$$
\operatorname{deg}\left(I-T_{\widetilde{\mu}}, \Omega \backslash B_{\varepsilon}(1), 0\right)=2 .
$$

For $\varepsilon>0$ small enough, there exists then a solution $u$ of $u-T_{\widetilde{\mu}}(u)=0$ in $\Omega \backslash B_{\varepsilon}(1)$, that is a non-constant positive bounded and $L$-periodic solution $u$ of (1.4) with the parameter $\widetilde{\mu}$ (remember that 1 is the only positive constant function solving (1.4)). The proof of Theorem 1.1 is thereby complete. 


\section{Bounds for the solutions of the Cauchy problem}

Here, we prove Theorem 1.2. We consider the Cauchy problem (1.6) with a parameter $\mu>0$ and a nonnegative initial condition $u_{0} \in L^{\infty}(\mathbb{R})$. We point out that we do not assume that $u_{0}$ is periodic. The maximum principle and standard parabolic estimates imply that the solution $u$ exists for all times $t \in(0,+\infty)$, is classical in $(0,+\infty) \times \mathbb{R}$ (and is even of class $C^{\infty}$ in this set) and satisfies

$$
0 \leq u(t, x) \leq e^{\mu t}\left\|u_{0}\right\|_{L^{\infty}(\mathbb{R})} \text { for every } t>0 \text { and } x \in \mathbb{R} .
$$

Our task is to improve (3.1) to a uniform in time estimate.

Let $\sigma>0$ be as in (1.3) and define the local average on the scale $\sigma$ :

$$
v(t, x)=\int_{x-\sigma / 2}^{x+\sigma / 2} u(t, y) d y \text { for }(t, x) \in[0,+\infty) \times \mathbb{R} .
$$

The function $v$ is of class $C^{\infty}((0,+\infty) \times \mathbb{R})$, continuous in $[0,+\infty) \times \mathbb{R}$ and satisfies a version of the upper bound (3.1):

$$
0 \leq v(t, x) \leq \sigma e^{\mu t}\left\|u_{0}\right\|_{L^{\infty}(\mathbb{R})} \text { for every }(t, x) \in[0,+\infty) \times \mathbb{R}
$$

Furthermore, the function $v$ obeys

$$
v_{t}(t, x)-v_{x x}(t, x)=\mu \int_{x-\sigma / 2}^{x+\sigma / 2} u(t, y)(1-(\phi * u)(t, y)) d y
$$

for every $(t, x) \in(0,+\infty) \times \mathbb{R}$, and, since the right-hand side of the above equation belongs to $L^{\infty}((a, b) \times \mathbb{R})$ for every $0 \leq a<b<+\infty$, the function $t \mapsto\|v(t, \cdot)\|_{L^{\infty}(\mathbb{R})}$ is actually continuous on $[0,+\infty)$.

Owing to (1.3), let now $\eta>0$ be such that

$$
\phi \geq \eta>0 \text { a.e. in }(-\sigma, \sigma)
$$

and let $M$ be any positive real number such that

$$
M>\max \left(\sigma\left\|u_{0}\right\|_{L^{\infty}(\mathbb{R})}, \frac{1}{\eta}\right)
$$

We will show that $\|v(t, \cdot)\|_{L^{\infty}(\mathbb{R})} \leq M$ for all $t>0$, by contradiction. Assume that this is false. Since $t \mapsto\|v(t, \cdot)\|_{L^{\infty}(\mathbb{R})}$ is continuous on $[0,+\infty)$, and

$$
\|v(0, \cdot)\|_{L^{\infty}(\mathbb{R})} \leq \sigma\left\|u_{0}\right\|_{L^{\infty}(\mathbb{R})}<M
$$

there exists $t_{0}>0$ such that $\left\|v\left(t_{0}, \cdot\right)\right\|_{L^{\infty}(\mathbb{R})}=M$ and $\|v(t, \cdot)\|_{L^{\infty}(\mathbb{R})}<M$ for all $t \in\left[0, t_{0}\right)$. Since $v$ is nonnegative, there exists then a sequence of real numbers $\left(x_{n}\right)_{n \in \mathbb{N}}$ such that $v\left(t_{0}, x_{n}\right) \rightarrow M$ as $n \rightarrow+\infty$. As usual, we define the translates

$$
u_{n}(t, x)=u\left(t, x+x_{n}\right) \text { and } v_{n}(t, x)=v\left(t, x+x_{n}\right) \text {, }
$$


for $n \in \mathbb{N}$ and $(t, x) \in(0,+\infty) \times \mathbb{R}$. From standard parabolic estimates, the sequences $\left(u_{n}\right)_{n \in \mathbb{N}}$ and $\left(v_{n}\right)_{n \in \mathbb{N}}$ are bounded in $C_{l o c}^{k}((0,+\infty) \times \mathbb{R})$ for every $k \in \mathbb{N}$ and converge in these spaces, up to extraction of a subsequence, to some nonnegative functions $u_{\infty}$ and $v_{\infty}$ of class $C^{\infty}((0,+\infty) \times \mathbb{R})$, such that

$$
v_{\infty}(t, x)=\int_{x-\sigma / 2}^{x+\sigma / 2} u_{\infty}(t, y) d y
$$

and

$$
\left(v_{\infty}\right)_{t}(t, x)=\left(v_{\infty}\right)_{x x}(t, x)+\mu \int_{x-\sigma / 2}^{x+\sigma / 2} u_{\infty}(t, y)\left(1-\left(\phi * u_{\infty}\right)(t, y)\right) d y
$$

for every $(t, x) \in(0,+\infty) \times \mathbb{R}$. The passage to the limit in the integral term is possible due to the local uniform convergence of $u_{n}$ to $u_{\infty}$ in $(0,+\infty) \times \mathbb{R}$ and to the local-in-time bounds (3.1). Furthermore, we have

$$
0 \leq v_{\infty}(t, x) \leq M
$$

for every $0<t \leq t_{0}$ and $x \in \mathbb{R}$, and $v_{\infty}\left(t_{0}, 0\right)=M$. Therefore, we have

$$
\left(v_{\infty}\right)_{t}\left(t_{0}, 0\right) \geq 0 \text { and }\left(v_{\infty}\right)_{x x}\left(t_{0}, 0\right) \leq 0
$$

whence,

$$
\int_{-\sigma / 2}^{\sigma / 2} u_{\infty}\left(t_{0}, y\right)\left(1-\left(\phi * u_{\infty}\right)\left(t_{0}, y\right)\right) d y \geq 0
$$

If

$$
\left(\phi * u_{\infty}\right)\left(t_{0}, \cdot\right)>1 \text { everywhere in }[-\sigma / 2, \sigma / 2],
$$

then the continuous function

$$
U=u_{\infty}\left(t_{0}, \cdot\right)\left(1-\left(\phi * u_{\infty}\right)\left(t_{0}, \cdot\right)\right)
$$

would be nonpositive on $[-\sigma / 2, \sigma / 2]$. Since its integral over $[-\sigma / 2, \sigma / 2]$ is nonnegative, the function $U$ would be identically equal to zero on $[-\sigma / 2, \sigma / 2]$. Moreover, it would then follow from (3.4) that $u_{\infty}\left(t_{0}, \cdot\right)=0$ on $[-\sigma / 2, \sigma / 2]$, whence $v_{\infty}\left(t_{0}, 0\right)=0$, contradicting the assumption that $v_{\infty}\left(t_{0}, 0\right)=M>0$. Therefore, there is a real number $y_{0} \in[-\sigma / 2, \sigma / 2]$ such that

$$
\left(\phi * u_{\infty}\right)\left(t_{0}, y_{0}\right) \leq 1
$$

Since both functions $\phi$ and $u_{\infty}$ are nonnegative, one gets from (3.2) that

$$
\begin{aligned}
1 \geq\left(\phi * u_{\infty}\right)\left(t_{0}, y_{0}\right) & \geq \int_{-\sigma}^{\sigma} \phi(y) u_{\infty}\left(t_{0}, y_{0}-y\right) d y \\
& \geq \eta \int_{-\sigma}^{\sigma} u_{\infty}\left(t_{0}, y_{0}-y\right) d y \geq \eta \int_{-\sigma / 2}^{\sigma / 2} u_{\infty}\left(t_{0}, y\right) d y=\eta v_{\infty}\left(t_{0}, 0\right)=\eta M .
\end{aligned}
$$

This contradicts the definition (3.3) of the constant $M$.

We conclude that $\|v(t, \cdot)\|_{L^{\infty}(\mathbb{R})} \leq M$ for all $t \geq 0$. Since $u$ is nonnegative, this means that

$$
0 \leq \int_{x-\sigma / 2}^{x+\sigma / 2} u(t, y) d y \leq M
$$


for every $t \geq 0$ and $x \in \mathbb{R}$. To get a global bound for $u$ itself, we just fix an arbitrary time $s \geq 1$ and infer from the maximum principle that

$$
0 \leq u(s, x) \leq w(s, x) \text { for every } x \in \mathbb{R} .
$$

Here, $w$ is the solution of the equation

$$
w_{t}=w_{x x}+\mu w
$$

with the initial condition at time $s-1$ given by $w(s-1, \cdot)=u(s-1, \cdot)$. It follows then from (3.5) that, for every $x \in \mathbb{R}$,

$$
0 \leq u(s, x) \leq e^{\mu} \int_{-\infty}^{+\infty} \frac{e^{-y^{2} / 4}}{\sqrt{4 \pi}} u(s-1, x-y) d y \leq \frac{2 M e^{\mu}}{\sqrt{4 \pi}} \sum_{k \in \mathbb{N}} e^{-k^{2} \sigma^{2} / 4}<+\infty .
$$

Together with the local-in-time bounds (3.1), this implies that $u$ is globally bounded. The proof of Theorem 1.2 is complete.

\section{Spreading speed for the Cauchy problem (1.6)}

This section is devoted to the proof of Theorem 1.3 on the asymptotic spreading speed for the solutions of the Cauchy problem (1.6). The proof of the upper bound (1.8) is immediate simply by comparing the solution $u$ of (1.6) to that of the (local) linear heat equation

$$
v_{t}=v_{x x}+\mu v \text {. }
$$

The proof of the lower bound (1.7) is more involved and, when compared to the proofs given in [3, 4, 5] for the analogous local problems, it requires additional arguments due to the nonlocality of (1.1), and the lack of comparison principle for the nonlocal equation (1.1). However, the maximum principle can be applied when $\phi * u$ is small and the equation (1.1) will then be compared in some suitable bounded boxes to a local linear equation close to (4.1).

\section{Proof of the upper bound}

We assume here that $u_{0}$ is compactly supported. Hence, there is $R>0$ such that $u_{0}(x)=0$ for a.e. $|x| \geq R$. Since $u(t, x) \geq 0$ for all $t>0$ and $x \in \mathbb{R}$, one has

$$
\mu u(t, x)(1-(\phi * u)(t, x)) \leq \mu u(t, x)
$$

for all $t>0$ and $x \in \mathbb{R}$. Let $v$ denote the solution of (4.1) for $t>0$ with initial condition $u_{0}$ at $t=0$. It follows from the maximum principle that $0 \leq u(t, x) \leq v(t, x)$ for all $t>0$ and $x \in \mathbb{R}$, whence

$$
0 \leq u(t, x) \leq \frac{e^{\mu t}}{\sqrt{4 \pi t}} \int_{-R}^{R} e^{-(x-y)^{2} /(4 t)} u_{0}(y) d y .
$$

Let $c$ be any arbitrary real number such that $c>2 \sqrt{\mu}$. For all $t \geq R / c$ and for all $|x| \geq c t$, one has

$$
0 \leq u(t, x) \leq \frac{e^{\mu t}\left\|u_{0}\right\|_{L^{\infty}(\mathbb{R})}}{\sqrt{4 \pi t}} \int_{-R}^{R} e^{-(c t-R)^{2} /(4 t)} d y,
$$


which immediately yields (1.8) (notice that, for every $t>0$, the maximum of $u(t, \cdot)$ on the set $(-\infty,-c t] \cup[c t,+\infty)$ is reached since $u(t, \cdot)$ is continuous, nonnegative, and converges to 0 at $\pm \infty$ from (4.2) $)$.

\section{Proof of the lower bound}

Assume for the sake of contradiction that the conclusion (1.7) does not hold. Then, since $u$ is nonnegative, there is $c$ such that

$$
0 \leq c<2 \sqrt{\mu}
$$

and there are two sequences $\left(t_{n}\right)_{n \in \mathbb{N}}$ in $(0,+\infty)$ and $\left(x_{n}\right)_{n \in \mathbb{N}}$ in $\mathbb{R}$ such that

$$
\left\{\begin{array}{l}
\left|x_{n}\right| \leq c t_{n} \text { for all } n \in \mathbb{N}, \\
t_{n} \rightarrow+\infty \text { and } u\left(t_{n}, x_{n}\right) \rightarrow 0 \text { as } n \rightarrow+\infty
\end{array}\right.
$$

We set

$$
c_{n}=\frac{x_{n}}{t_{n}} \in[-c, c]
$$

Up to extraction of a subsequence, one can assume that $c_{n} \rightarrow c_{\infty} \in[-c, c]$ as $n \rightarrow+\infty$.

Furthermore, for every $n \in \mathbb{N}$ and $(t, x) \in\left(-t_{n},+\infty\right) \times \mathbb{R}$, we define the shifted functions

$$
u_{n}(t, x)=u\left(t+t_{n}, x+x_{n}\right)
$$

From Theorem 1.2, the sequence $\left(\left\|u_{n}\right\|_{L^{\infty}\left(\left(-t_{n},+\infty\right) \times \mathbb{R}\right)}\right)_{n \in \mathbb{N}}$ is bounded. Therefore, the standard parabolic estimates imply that the functions $u_{n}$ converge in $C_{l o c}^{1,2}(\mathbb{R} \times \mathbb{R})$, up to extraction of a subsequence, to a classical bounded solution $u_{\infty}$ of

$$
\left(u_{\infty}\right)_{t}=\left(u_{\infty}\right)_{x x}+\mu u_{\infty}\left(1-\phi * u_{\infty}\right) \text { in } \mathbb{R} \times \mathbb{R},
$$

such that $u_{\infty} \geq 0$ in $\mathbb{R} \times \mathbb{R}$ and $u_{\infty}(0,0)=0$. By viewing $\mu\left(1-\phi * u_{\infty}\right)$ as a coefficient in $L^{\infty}(\mathbb{R} \times \mathbb{R})$, it follows from the strong parabolic maximum principle and the uniqueness of the solutions of the Cauchy problem that $u_{\infty}(t, x)=0$ for all $(t, x) \in \mathbb{R} \times \mathbb{R}$ (the limit $u_{\infty}$ being unique, one can then infer that the whole sequence $\left(u_{n}\right)_{n \in \mathbb{N}}$ converges to 0 in $\left.C_{l o c}^{1,2}(\mathbb{R} \times \mathbb{R})\right)$. As a consequence, the nonnegative functions $v_{n}$ defined in $\left(-t_{n},+\infty\right) \times \mathbb{R}$ by

$$
v_{n}(t, x)=u_{n}\left(t, x+c_{n} t\right)=u\left(t+t_{n}, x+c_{n}\left(t+t_{n}\right)\right)
$$

converge to 0 locally uniformly in $\mathbb{R} \times \mathbb{R}$, due to the boundedness of the speeds $c_{n}$ defined in (4.4). Hence the nonnegative functions $\phi * v_{n}$ also converge to 0 locally uniformly in $\mathbb{R} \times \mathbb{R}$, since the sequence $\left(\left\|v_{n}\right\|_{L^{\infty}\left(\left(-t_{n},+\infty\right) \times \mathbb{R}\right)}\right)_{n \in \mathbb{N}}$ is bounded.

Let us now fix some parameters which are independent of $n$. First, let $\delta>0$ be such that

$$
\mu(1-\delta) \geq \frac{c^{2}}{4}+\delta,
$$

and also let $R>0$ be such that

$$
\frac{\pi^{2}}{4 R^{2}} \leq \delta
$$


Since $u(1, \cdot)$ is continuous from parabolic regularity, and is positive in $\mathbb{R}$ from the strong parabolic maximum principle, there is $\eta>0$ such that

$$
u(1, x) \geq \eta>0 \text { for all }|x| \leq R+c .
$$

Without loss of generality, one can assume that $t_{n}>1$ for every $n \in \mathbb{N}$. Since $\phi * v_{n} \rightarrow 0$ locally uniformly in $\mathbb{R} \times \mathbb{R}$ as $n \rightarrow+\infty$, for $n$ sufficiently large $(n \geq N)$ we may define

$$
t_{n}^{*}=\inf \left\{t \in\left[-t_{n}+1,0\right] ;(0 \leq) \phi * v_{n} \leq \delta \text { in }[t, 0] \times[-R, R]\right\}, \quad n \geq N,
$$

with $\delta$ and $R$ as in (4.5) and (4.6), and we may assume that $t_{n}^{*}<0$. Furthermore, for every $n \geq N$, by continuity of $\phi * v_{n}$ in $\left(-t_{n},+\infty\right) \times \mathbb{R}$, the infimum is a minimum in the definition of $t_{n}^{*}$ and

$$
0 \leq \phi * v_{n} \leq \delta \text { in }\left[t_{n}^{*}, 0\right] \times[-R, R]
$$

On the other hand, we have

$$
v_{n}\left(-t_{n}+1, x\right)=u\left(1, x+c_{n}\right) \geq \eta \text { for all }|x| \leq R,
$$

from (4.4) and (4.7), for all $n \in \mathbb{N}$. Therefore, again by continuity of $\phi * v_{n}$ in $\left(-t_{n},+\infty\right) \times \mathbb{R}$ and by minimality of $t_{n}^{*}$, for each $n \geq N$ there is the following dichotomy:

$$
\begin{cases}\text { either } & \left(t_{n}^{*}>-t_{n}+1 \text { and } \max _{[-R, R]}\left(\phi * v_{n}\right)\left(t_{n}^{*}, \cdot\right)=\delta\right) \\ \text { or } \quad\left(t_{n}^{*}=-t_{n}+1 \text { and } \min _{[-R, R]} v_{n}\left(t_{n}^{*}, \cdot\right) \geq \eta\right) .\end{cases}
$$

Next, we claim that there exists $\rho>0$ such that

$$
\min _{[-R, R]} v_{n}\left(t_{n}^{*}, \cdot\right) \geq \rho>0 \text { for all } n \geq N
$$

Note that this claim would be immediate if the second assertion of (4.9) always holds, but there is a priori no reason for the first assertion not to hold. Remember that $\min _{[-R, R]} v_{n}\left(t_{n}^{*}, \cdot\right)>0$ for each fixed $n \geq N$. So, if (4.10) were not true, then, up to extraction of a subsequence, there would exist a sequence of points $\left(y_{n}\right)_{n \geq N}$ in $[-R, R]$ such that

$$
v_{n}\left(t_{n}^{*}, y_{n}\right) \rightarrow 0 \text { and } y_{n} \rightarrow y_{\infty} \in[-R, R] \text { as } n \rightarrow+\infty .
$$

We will use the translates

$$
w_{n}(t, x)=v_{n}\left(t+t_{n}^{*}, x\right),
$$

defined for all $n \geq N$ and $(t, x) \in\left(-t_{n}-t_{n}^{*},+\infty\right) \times \mathbb{R}$. Since the functions $v_{n}$ solve

$$
\left(v_{n}\right)_{t}=\left(v_{n}\right)_{x x}+c_{n}\left(v_{n}\right)_{x}+\mu v_{n}\left(1-\phi * v_{n}\right) \text { in }\left(-t_{n},+\infty\right) \times \mathbb{R}
$$

the functions $w_{n}$ solve the same equation, in $\left(-t_{n}-t_{n}^{*},+\infty\right) \times \mathbb{R}$. Notice that $-t_{n}-t_{n}^{*} \leq-1$ for all $n \geq N$, that $c_{n} \rightarrow c_{\infty}$ as $n \rightarrow+\infty$, that the functions $w_{n}$ are all nonnegative and that the

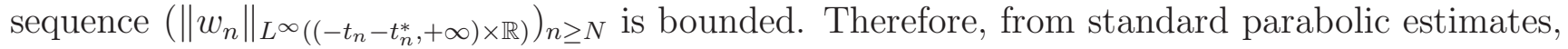


the functions $w_{n}$ converge in $C_{l o c}^{1,2}((-1,+\infty) \times \mathbb{R})$, up to extraction of a subsequence, to a classical bounded solution $w_{\infty}$ of

$$
\left(w_{\infty}\right)_{t}=\left(w_{\infty}\right)_{x x}+c_{\infty}\left(w_{\infty}\right)_{x}+\mu w_{\infty}\left(1-\phi * w_{\infty}\right) \text { in }(-1,+\infty) \times \mathbb{R},
$$

such that

$$
w_{\infty}(t, x) \geq 0 \text { for all }(t, x) \in(-1,+\infty) \times \mathbb{R} \text { and } w_{\infty}\left(0, y_{\infty}\right)=0 .
$$

It follows form the strong maximum principle and the uniqueness of the solutions of the Cauchy problem that

$$
w_{\infty}(t, x)=0 \text { for all }(t, x) \in(-1,+\infty) \times \mathbb{R} .
$$

In other words, $w_{n} \rightarrow 0$ as $n \rightarrow+\infty$ (at least) locally uniformly in $(-1,+\infty) \times \mathbb{R}$, whence

$$
\phi * w_{n} \rightarrow 0 \text { as } n \rightarrow+\infty,
$$

locally uniformly in $(-1,+\infty) \times \mathbb{R}$ by boundedness of the sequence $\left(\left\|w_{n}\right\|_{L^{\infty}((-1,+\infty) \times \mathbb{R})}\right)_{n \geq N}$. Therefore, we have

$$
v_{n}\left(t_{n}^{*}, \cdot\right) \rightarrow 0 \text { and }\left(\phi * v_{n}\right)\left(t_{n}^{*}, \cdot\right) \rightarrow 0 \text { locally uniformly in } \mathbb{R} \text { as } n \rightarrow+\infty \text {. }
$$

This is a contradiction to (4.9), since both $\delta$ and $\eta$ are positive. Therefore, claim (4.10) is proved.

Now, (4.8), (4.10) and (4.11) imply that we have the following situation: for every $n \geq N$, one has $-t_{n}+1 \leq t_{n}^{*}<0$ and, in the box $\left[t_{n}^{*}, 0\right] \times[-R, R]$, the nonnegative function $v_{n}$ satisfies

$$
\left\{\begin{aligned}
\left(v_{n}\right)_{t} & =\left(v_{n}\right)_{x x}+c_{n}\left(v_{n}\right)_{x}+\mu v_{n}\left(1-\phi * v_{n}\right) & & \\
& \geq\left(v_{n}\right)_{x x}+c_{n}\left(v_{n}\right)_{x}+\mu(1-\delta) v_{n} & & \text { in }\left[t_{n}^{*}, 0\right] \times[-R, R] \\
v_{n}(t, \pm R) & \geq 0 & & \text { for all } t \in\left[t_{n}^{*}, 0\right], \\
v_{n}\left(t_{n}^{*}, x\right) & \geq \rho & & \text { for all } x \in[-R, R] .
\end{aligned}\right.
$$

On the other hand, for every $n \geq N$, the function $\psi_{n}$ defined in $[-R, R]$ by

$$
\psi_{n}(x)=\rho e^{-c_{n} x / 2-c R / 2} \cos \left(\frac{\pi x}{2 R}\right)
$$

satisfies $0 \leq \psi_{n} \leq \rho$ in $[-R, R]$ from (4.4), $\psi_{n}( \pm R)=0$ and

$$
\psi_{n}^{\prime \prime}+c_{n} \psi_{n}^{\prime}+\mu(1-\delta) \psi_{n}=\left(\mu(1-\delta)-\frac{c_{n}^{2}}{4}-\frac{\pi^{2}}{4 R^{2}}\right) \psi_{n} \geq 0 \text { in }[-R, R]
$$

from our choice of $\delta$ and $R$ : see (4.4), (4.5) and (4.6). In other words, the time-independent function $\phi_{n}$ is a subsolution for the problem (4.12) satisfied by $v_{n}$ in $\left[t_{n}^{*}, 0\right] \times[-R, R]$. It follows then from the parabolic maximum principle that

$$
v_{n}(t, x) \geq \phi_{n}(x) \text { for all }(t, x) \in\left[t_{n}^{*}, 0\right] \times[-R, R],
$$

and for all $n \geq N$. In particular,

$$
u\left(t_{n}, x_{n}\right)=v_{n}(0,0) \geq \phi_{n}(0)=\rho e^{-c R / 2} \text { for all } n \geq N .
$$

However, assumption (4.3) means that $u\left(t_{n}, x_{n}\right) \rightarrow 0$ as $n \rightarrow+\infty$, while $\rho e^{-c R / 2}>0$ from the positivity of $\rho$ in (4.10). One has then reached a contradiction.

Therefore, (1.7) holds and the proof of Theorem 1.3 is complete. 


\section{References}

[1] M. Alfaro and J. Coville, Rapid traveling waves in the nonlocal Fisher equation connect two unstable states, Appl. Math. Lett. 5, 2012, 2095-2099.

[2] N. Apreutesei, N. Bessonov, V. Volpert, and V. Vougalter, Spatial structures and generalized travelling waves for an integro-differential equation, Disc. Cont. Dyn. Syst. B 13, 2010, 537-557.

[3] D.G. Aronson and H.F. Weinberger, Multidimensional nonlinear diffusions arising in population genetics, Adv. Math. 30, 1978, 33-76.

[4] H. Berestycki, F. Hamel, and G. Nadin, Asymptotic spreading in heterogeneous diffusive media, J. Funct. Anal. 255, 2008, 2146-2189.

[5] H. Berestycki, F. Hamel, and N. Nadirashvili, The speed of propagation for KPP type problems. II - General domains, J. Amer. Math. Soc. 23, 2010, 1-34.

[6] H. Berestycki, G. Nadin, B. Perthame, and L. Ryzhik, The non-local Fisher-KPP equation: traveling waves and steady states, Nonlinearity 22, 2009, 2813-2844.

[7] H. Berestycki, L. Nirenberg, and S.R.S. Varadhan, The principal eigenvalue and maximum principle for second order elliptic operators in general domains, Comm. Pure Appl. Math. 47, 1994, 47-92.

[8] N.F. Britton, Spatial structures and periodic travelling waves in an integro-differential reaction-diffusion population model, SIAM J. Appl. Math. 50, 1990, 1663-1688.

[9] J. Fang and X.-Q. Zhao, Monotone wavefronts of the nonlocal Fisher-KPP equation. Nonlinearity 24, 2011, 3043-3054.

[10] R.A. Fisher, The advance of advantageous genes, Ann. Eugenics 7, 1937, 335-369.

[11] J.-E. Furter and M. Grinfeld, Local vs. nonlocal interactions in population dynamics, J. Math. Biol. 27, 1989, 65-80.

[12] S. Genieys, V. Volpert, and P. Auger, Pattern and waves for a model in population dynamics with nonlocal consumption of resources, Math. Model. Nat. Phenom. 1, 2006, $65-82$.

[13] S. Gourley, Traveling front solutions of a nonlocal Fisher equation, J. Math. Biol. 41, 2000, 272-284.

[14] S.A. Gourley and N.F. Britton, Instability of travelling wave solutions of a population model with nonlocal effects, IMA J. Appl. Math. 51, 1993, 299-310.

[15] A.N. Kolmogorov, I.G. Petrovsky, and N.S. Piskunov, Étude de l'équation de la diffusion avec croissance de la quantité de matière et son application à un problème biologique, Bull. Univ. État Moscou, Sér. Intern. A 1, 1937, 1-26. 
[16] G. Nadin, B. Perthame, and M. Tang, Can a traveling wave connect two unstable states? The case of the nonlocal Fisher equation, C. R. Math. Acad. Sci. Paris 349, 2011, 553-557.

[17] G. Nadin, B. Perthame, L. Rossi, and L. Ryzhik, Wave-like solutions for nonlocal reactiondiffusion equations: a toy model, Math. Model. Nat. Phenom. 8, 2013, 33-41.

[18] Z.-C. Wang, W.-T. Li, and S. Ruan, Travelling wave fronts of reaction-diffusion systems with spatio-temporal delays, J. Diff. Eq. 222, 2006, 185-232. 